\title{
A Cluster of Friedreich's Ataxia in Rimouski, Québec
}

\author{
J.P. BOUCHARD, A. BARBEAU, R. BOUCHARD, M. PAQUET AND R. W. BOUCHARD
}

SUMMARY: We described a cluster of 8 independent sibships of Friedreich's ataxia in the St-Fabien parish of Rimouski and have shown that they are all related within 6 generations. The siudy of this geographic and genetic isolate permitted the investigation of certain unusual features of the disease such as constant myopia, delayed reaction times to pain. flexor spasms, and a rapid evolution.

RÉSUMÉ: Nous décrivons un groupement de 8 fratries indépendantes d'ataxie de Friedreich dans la région de St-Fabien de Rimouski, et montrons qu'elles sont toutes reliées en dedans de 6 générations. L'étude de cet isolat génétique et géographique permet l'investigation de certains aspects inhabituels de la maladie, tels la présence constante d'une myopie, la lenteur des temps de réaction aux stimuli douloureux, les spasmes de flexion et l'évolution plus rapide.

From l'Hôpital de l'Enfant-Jésus, Quebec City: I'Institut de Cardiologie de Québec and the Clinical Research Institute of Montreal.

Reprint requests for the complete supplement on Friedreich's Ataxia (Phase Two, Part Two) to:

Dr. André Barbeau, Clinical Research Institute of Montreal, 110 Pine Avenue West, Montreal, Quebec, Canada, H2W IR7.

\section{INTRODUCTION}

The renewed interest in the study of hereditary ataxias in Quebec in the last five years led first to the characterization of "classical" cases (Geoffroy et al., 1976). In that investigation of 50 patients, almost one third (14) were "atypical" on clinical and electrophysiological grounds (Peyronnard et al., 1976). Later, we identified a large group of patients (almost one hundred patients) presenting with autosomal recessive spastic ataxia in the Charlevoix and Saguenay area (Bouchard et al., 1978) and with electrophysiological findings similar to those of the atypical group in the first study (Peyronnard et al., 1976; Bouchard et al., 1979, this issue).

Recently, we had the opportunity to study a group of nineteen ataxic patients from another region of Quebec, clustered within 8 families of which at least seven are closely related. A few generations prior to the study, the ancestors were living in a small village, founded in 1828, and most of their descendants are still inhabiting Rimouski county on the south shore of the St-Laurent River, two hundred miles below Quebec City. The population of the county was around 65,000 in 1976.

\section{Genealogical Studies}

Because church records are complete in the Province of Quebec, it has been possible to trace most of the involved families who all lived within 25 miles of the original village of St. Fabien de Rimouski. As can be seen in Fig. 1, seven present sibships with different surnames can be traced to a single ancestral couple, through direct descent of probable carriers. There are many consanguinous marriages in this family and we are still trying to decipher the other probable carrier lines. The studies, to date, are sufficiently advanced to suggest that we are dealing with both a genetic and a geographical isolate. Such groupings are extremely important to unravel the respective influences of gene pools, environment, and other extrinsic factors.

\section{Clinical Findings}

It is known that hereditary ataxias encompass a number of variants, often creating a special form in many kindreds. Besides the numerous "intermediate and atypical forms", large groups of "classical" patients are linked genetically and also demonstrate features that contribute to the spectrum of abnormalities seen in spinocerebellar degenerations (Geoffroy et al., 1976; Tyrer, 1975). The group presented here underlines the fact that geographical and genetic isolation may produce significant differences from the general picture, without amputating the accepted list of symptoms and signs of Friedreich's ataxia. Such groups, with little inter and intra familial differences, may help to a better understanding of certain peculiar clinical aspects too diffuse in other kinships.

We know of and we have examined nineteen patients with the full blown disease in the Rimouski area. (A young girl of six has no symptom of ataxia and is not tabulated with the group. She exhibited areflexia and severe cardiomyopathy. Three other patients were dead at the time of the study.) There are 8 males and 11 females: ages ranges from 14 to 24 (mean 18.62) for males and from 14 to 34 (mean 23.27) for females for a global mean of 21.31. Of this group, five patients under 18 years of age can still walk. Ten patients were admitted to hospital and underwent the protocol investigations of the Quebec 
FRIEDREICH'S ATAXIA ISOLATE IN ST-FABIEN DE RIMOUSKI, QUEBEC

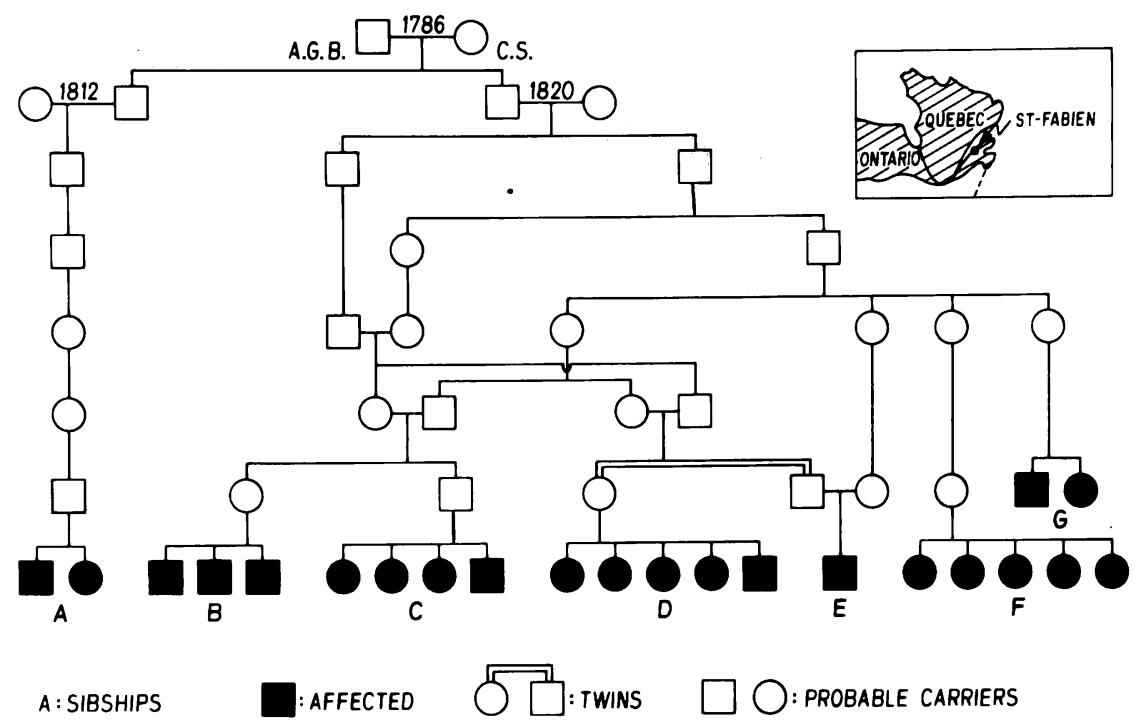

Figure 1 -In this diagram we traced one direct line of probable carriers to a common ancestral couple who married in 1786. Seven sibships can be traced. This diagram is simplified from the more complex one which would trace all possible carrier lines through the various consanguinous marriages. In the affected sibships, for the purpose of clarity, only affected children are indicated.

Cooperative Study. There were four males ( 18 to 20 year old, mean of 19.5 ) and 6 females (17 to 28 years of age, mean of 23.0). In this group the onset of ataxia was between 5 and 8 years of age. The disease then progressed steadily and the patients were usually in wheelchairs in their mid-teens. In one family there was some variation in the age of onset from 6 to 12 and less homogeneity in clinical signs. Early onset is not rare in Friedreich's ataxia (Tyrer, 1975; Geoffroy, 1976), but here it shows a strong familial similarity. The aim of this report is not to repeat and check on the long list of accepted symptoms and signs in Friedreich's ataxia. Rather, we would like to underline less common characteristics of this group, also reported occasionally in other kinships with the disease. Data are given for the ten hospitalized patients who completed the protocol. Ataxia of gait was always the first sign, but truncal ataxia soon became so marked that sitting was impossible. None of the 10 patients in hospital could remain sitting without holding the sides of the bed. Dysarthria was variable, often marked, and nystag- mus was discrete in half the cases. Increased saccadic pursuit was noted in 2 . The only eye problem noted was slight myopia in 9 of 10 , symmetrical and varying between -1 and -3 diopters. Fundi and color vision were normal.

Spasticity in the lower limbs was exemplified by Babinski signs in 8 of 10 patients and by spontaneous or elicited flexor spasms in 6. Eight subjects complained of cramps in various muscles. The cutaneous abdominal reflexes were present in half the cases while deep tendon reflexes were abolished. The dissociation between the apparent flaccidity of the legs and the marked corticospinal dysfunction leading to flexor spasms has received attention from Tyrer (1975) and seems to be a rare finding.

Seven of the 10 patients presented with bladder problems, either urgency, stress incontinence, or frequent accidents. Dysphagia, especially for liquids, was more frequent affecting 9 of 10 patients. Gastric pain and vomiting, with normal gastrointestinal investigations, were prominent in one case. These symptoms have been reported rarely (Bell and Carmichael, 1939; Tyrer, 1975) and are attributed to autonomic dysfunction.

Most important were the sensory deficits. A definite decrease in proprioception was demonstrated by the absence of vibration sense at the toes and absence or decrease at the ankle, often asymmetrical. Position sense was also impaired in the toes in all cases but one. In two patients it was abnormal at the ankle and finger levels. Superficial sensory deficits were also present in most cases $(7 / 10)$, consisting mostly of long delays between stimulus and perception of pain (up to 2 seconds) which then often provoked flexor spasms. Temperature perception was clearly impaired in one case, a 19 year old male in a wheelchair for 6 years who showed no perception of vibrations to the waist and marked delays in pain perception.

\section{Radiological Evaluation}

Scoliosis was present in all cases, slight in one $\left(<20^{\circ}\right)$, moderate (20$\left.40^{\circ}\right)$ in four, and severe $\left(>40^{\circ}\right)$ in five, up to $105^{\circ}$ in the youngest patient admitted to hospital. Pes cavus was seen in most cases, but in some patients with no active distal movements only $\mathrm{X}$-rays could clearly show the deformity. The neuroradiological evaluation is described elsewhere and indicates signs of superior vermis and anterior cerebellar lobe atrophy.

\section{Cardiovascular evaluation}

Eight of the ten patients evaluated were asymptomatic. Two patients with known hypertrophic cardiomyopathy were taking regular propanolol because of incapacitating symptoms: angina in one, and marked dyspnea and easy fatigability in the other. Arterial blood pressure was normal in all patients. Cardivascular examination was entirely normal in 3 patients. Two patients presented with an early systolic click at the apex. Two additional patients had a systolic ejection murmur at the left sternal border. One patient had an irregular rhythm which was due to the presence of numerous atrial premature contractions.

Radiological evaluation of the heart was rendered difficult by the presence 
of a marked scoliosis in most of the patients. In all cases where the $\mathrm{c} / \mathrm{t}$ ratio could be evaluated, it was within normal limits. One patient had radiological evidence of left ventricular dilatation.

The ECG was normal in one patient. The remaining 9 patients presented the following ECG anomalies: inferolateral ischemia in 4, septal hypertrophy in 2, right ventricular hypertrophy in 2, left axis deviation, atrial premature contraction and diffuse non specific anomalies of the repolarization phase in one case each. Echocardiographic examination was performed in 9 out of the 10 patients. The tracing was normal in two. Pansystolic prolapse of the mitral valve was observed in 3 cases. Left ventricular wall hypertrophy was present in 6 cases; it was of the concentric type in 3 while in the remaining 3 the echocardiogram showed asymmetric septal hypertrophy (ASH).

The 10 patients included in this study presented a wide variety of cardiovascular anomalies. The findings on physical examination were nonspecific in all but one patient who had auscultatory evidence of a subaortic obstruction. Ischemia in the infero-lateral region was the most common electrocardiographic findings $(4 / 10)$; this may be secondary to poor perfusion of the sub-endocardial layers of the myocardium in the presence of left ventricular wall hypertrophy. This finding, either of the asymmetric variety or of the concentric type was the most common echocardiographic anomaly noted in this series. This is in accordance with the findings of Gattiker et al. (1976). None of the patients had signs of left ventricular outflow tract obstruction i.e. systolic anterior motion (SAM) of the anterior mitral leaflet or midsystolic closure of the aortic valve. Mitral valve prolapse was observed in three patients.

While most patients presented with few if any abnormalities on physical examination, the ECG and the echocardiogram were abnormal in most cases and seem to be better indicators of the evolution of the cardiac involvement than the subjective complaints and the physical examination, as was previously noted in Phase One. Furthermore, our findings are in agreement with what has been reported (Boyer et al., 1962) in that there is no correlation between the degree of neurologic impairment and the severity of the cardiac disease.

\section{Psychometric evaluation}

The Ottawa-Wechsler intelligence battery was administered to all ten patients when in hospital. The patients were clinically observed while performing, specifically with respect to the degree of physical handicap and its possible influence on the results of different tests.

The mean $z$-scores (mean equals 0 and standard deviation equals 1 for the entire population) for each scale are presented in Table 1 with the t-test for paired data between both scales. Our patients had normal scoring for verbal scale (mean I.Q.: 100.80; SD: 11.56) but scored significantly lower $(\mathrm{P}>.01)$ on the non-verbal scale (mean I.Q.: 81.55 ; SD: 13.43). Only picture completion with a mean z-score of .03 was normal in that scale. The worst performance was with digit symbol (mean z-score: -2.19) and this is related partly to the clumsiness or the weakness of the patients' hands in a timed test. The lower performance on the other sub-tests could not be accounted for on this basis according to our clinical observations during the tests.

As a group, our patients scored better on both scales than another clinically different ataxic group recently studied (Bouchard et al., 1978), although the former were much more clumsy. This supports the view that physical disability cannot in itself explain all the low scores. If one excludes the digit symbol performance, the non-verbal profile still remains slightly lower than normal, although it now approaches the lower limits of the normal population. A greater number of patients would probably confirm this lower profile and better define its significance. There was no difference for either scale between males and females. Geoffroy et al. (1976) also found in their series a verbal I.Q. in the lower limits of normal, but females scored significantly lower. They did not report the non-verbal results, claiming their patients were too severely disabled to allow conclusions. Davies (1949) found in 18 patients a verbal WechslerBellevue I.Q. close to normal (ranging between 75 and 117); for two other tests (the Mill Hill vocabulary test and a non-verbal test, the Raven's progressive matrices). Davies com-

TABLE 1

Means of each Ottawa-Wechsler Scale adjusted for Age, and $T$-Test for Paired Data between Verbal and Non-Verbal I.Q. ( $N: 10)$

\begin{tabular}{lcc}
\hline Verbal Scale & $\begin{array}{c}\text { Mean } \\
(z-s c o r e)\end{array}$ & \\
\hline Information & -.50 & \\
Comprehension & .47 & VERBAL I.Q.* \\
Digit Span & .24 & MEAN: 100.80 \\
Arithmetic & -.80 & S.D.: 11.56 \\
Similarities & .70 & \\
& & \\
\hline Non-Verbal Scale & & \\
\hline & & \\
Picture Arrangement & -.35 & NON-VERBAL I.Q.* \\
Picture Completion & .03 & MEAN: 81.55 \\
Block Design & -.94 & S.D.: 13.43 \\
Object Assembly & -.98 & \\
Digit Symbol & -2.19 & \\
\hline
\end{tabular}

$* t: 11.49 ; \quad p<.01$ 
pared his patients to a group of chronic invalids (non-neurological diseases) and found no significant differences. These studies, like ours, confirm that Friedreich's ataxia patients have an overall average verbal intelligence. Our study suggests that these patients have difficulties with non-verbal materials that are not due to their physical disability alone.

\section{Electrophysiological studies}

Detailed results of electroencephalography, electromyography, and nerve conduction studies are given in other papers in this issue. These results were identical to the data previously published on Friedreich's ataxia.

\section{Laboratory results}

Hematologic data were normal for all patients, as well as the results of folate and vitamin $B_{12}$ determinations done with the kits of Bio-Rad Laboratories (Quanta-Count Folate Radioassay and Quanta-Count $\mathrm{B}_{12}$ Radioassay). Serum cholesterol and triglycerides levels were determined in another laboratory (A.D.) and were normal. Fasting blood glucose levels were normal and intravenous glucose tolerance tests failed to show any significant difference from normal.

Determination of total bilirubin was done on three consecutive days for the patients admitted to hospital, and for most patients of the whole group and their parents in a subsequent local visit in the field. Total bilirubinemia was slightly increased in three patients from 3 families and a similar increase was present in one parent.

Cerebrospinal fluid (CSF) was obtained by lumbar puncture in nine patients and showed normal cellular content. Total CSF proteins were normal and ranged from 13 to $43 \mathrm{mg} \%$ (mean 27.8). Electrophoresis did not disclose abnormal patterns in any of the cases.

\section{CONCLUSIONS}

The clinical picture of "typical" Friedreich's ataxia has been reviewed by Tyrer (1975) and by Geoffroy et al. (1976) who listed the primary and secondary symptoms and signs of the disease. Our patients conform to all these criteria for Friedreich's ataxia.

The homogeneous group that we have described issued from common ancestors and demonstrated almost universal myopia, flexor spasms, and delay in pain perception. Liquid dysphagia, urinary urgency, and incontinence were frequent and could have been underestimated in other series. Scoliosis, cardiac involvement, and electrophysiological studies confirmed the diagnosis. Psychometric evaluations showed better result than expected in non-verbal tests, despite the severe motor deficit.

Laboratory tests failed to disclose any diabetes mellitus in this group, but familial hyperbilirubinemia was found again in $37 \%$ of the families and one parent showed an increased value. Although no direct link can be seen between the dominantly transmitted Gilbert's disease and the autosomal recessive ataxias in Quebec (Friedreich's ARSACS), a 30 to $40 \%$ correlation has been found throughout this cooperative study.

The more "spinal" form of Friedreich's ataxia that we have reported here, demonstrated rare manifestations of spasticity (flexor spasm), sensory involvement (delayed pain perception), and a somewhat more severe course. Further investigation of these peculiarities may shed more light on the pathogenesis of the spino-cerebellar degenerative diseases. Such studies on geographical and genetic isolates will be more difficult in Quebec in the future, as in other parts of the world (Refsum, 1978).

\section{ACKNOWLEDGEMENTS}

The authors would like to express their thanks to Abbé Grégoire Riou for his help in the genealogical studies.

\section{REFERENCES}

BARBEAU, A., LESIEGE, M., BRETON, G., COALLIER, R. and BOUCHARD, J. P. (1976). Friedreich's ataxia: preliminary results of some genealogical research. Can. J. Neurol. Sci. 4: 303-306.

BELL, J. M. and CARMICHAEL, E. A. (1939). On hereditary ataxia and spastic paraplegia. In: Treasury of Human Inheritance, Cambridge Press, London, Vol. 4, pp. 141-284.

BOUCHARD, J. P., BARBEAU, A., BOUCHARD, R. W. (1978). Autosomal recessive spastic ataxia of CharlevoixSaguenay (ARSACS). Can. J. Neurol. Sci. 5: 61-69.

BOYER, S. H., CHISHOLM, A. W. and McKUSICK, V. A. (1962). Cardiac aspects of Friedreich's ataxia. Circulation 25: 493505.

DAVIES, D. L. (1949). The intelligence of patients with Friedreich's ataxia. J. Neurol. Neurosurg. Psychiat. 12: 34-38.

GATTIKER, H. F., DAVIGNON, A., BOZIO, A., BATTLE-DIAZ, J., GEOFFROY, G., LEMIEUX, B. and BARBEAU, A. (1976). Echocardiographic findings in Friedreich's ataxia. Can. J. Neurol. Sci. 4: 329-332.

GEOFFROY, G., BARBEAU, A., BRETON, G., LEMIEUX, B., AUBE, M., LEGER, C. and BOUCHARD, J. P. (1976). Clinical description and Roentgenologic evaluation of patients with Friedreich's ataxia. Can. J. Neurol. Sci. 4: 279-286.

PEYRONNARD, J. M., BOUCHARD, J. P., LAPOINTE, L., LAMONTAGNE, A., LEMIEUX, B. and BARBEAU, A. (1976). Nerve conduction studies and electromyography in Friedreich's ataxia. Can. J. Neurol. Sci. 4: 313-317.

REFSUM, S. and SKRE, H. (1978). Nosology, genetics and epidemiology of hereditary ataxias, with particular reference to the epidemiology of these disorders in Western Norway. In: Advances in Neurology, Vol. 19, B.S. Schoenberg, editor. Raven Press, New York, pp. 497-508.

TYRER, J. H. (1975). Friedreich's ataxia. In: Handbook of clinical neurology. P. J. Vinken and G. W. Bruyn, editors. North Holland Publ. Co., Amsterdam. Vol. 21, pp 319-364. 